\title{
OPEN-SET EVOLVING ACOUSTIC SCENE CLASSIFICATION SYSTEM
}

\author{
Fatemeh Saki, Yinyi Guo, Cheng-Yu Hung \\ Lae-Hoon Kim, Manyu Deshpande, Sunkuk Moon, Eunjeong Koh, and Erik Visser
}

Qualcomm Technologies, Inc., San Diego, USA

\begin{abstract}
Most audio recognition/classification systems assume a static and closed-set model, where training and testing data are drawn from a prior distribution. However, in real-world audio recognition/classification problems, such a distribution is unknown, and training data is limited and incomplete at training time. As it is difficult to collect exhaustive training samples to train classifiers. Datasets at prediction time are evolving and the trained model must deal with an infinite number of unseen/unknown categories. Therefore, it is desired to have an open-set classifier that not only accurately classifies the known classes into their respective classes but also effectively identifies unknown samples and learns them. This paper introduces an open-set evolving audio classification technique, which can effectively recognize and learn unknown classes continuously in an unsupervised manner. The proposed method consists of several steps: a) recognizing sound signals and associating them with known classes while also being able to identify the unknown classes; b) detecting the hidden unknown classes among the rejected sound samples; c) learning those novel detected classes and updating the classifier. The experimental results illustrate the effectiveness of the developed approach in detecting unknown sound classes compared to extreme value machine (EVM) and Weibull-calibrated SVM (W-SVM).
\end{abstract}

Index Terms-Acoustic scene classification, open-set recognition, support vector data description

\section{INTRODUCTION}

Research on acoustic scene classification (ASC) has been receiving increased attention over the past decade, which has led to a considerable amount of new sound modeling and recognition techniques. ASC plays a major role in machine hearing systems. Where, the primary goal is achieving human-like auditory recognition of ambient sound signals [1, 2]. Some example applications include contextaware devices that automatically adjust their operation mode according to surrounding sounds, such as hearing aid devices that their speech enhancement parameters are adjusted depending on the background noise type [3]. Some other applications are robotics [4], monitoring elderly people, and acoustic monitoring systems in smart homes for detecting events such as glass breaking, baby crying, and gunshot [5].
One limitation of the existing ASC systems is their closed-set nature, that is a fixed and limited number of known classes are used during the training. In closed-set classifiers, it is assumed that during test time, the test data is drawn from the same set of classes as the training data. This guarantees that every input samples are classified into exactly one of the training classes. However, most applications for ASCs in nature are open-set problems. In other words, in an open-set framework, the test data could include samples associated with unknown classes as well. Therefore, it is necessary for an ASC to detect if a sound signal is associated with an unknown category. It is also desired to learn the unknown instances that appear more frequently. To our knowledge, the following contributions are the only existing ASC systems that partially implement the open-set framework. In [6] an open-set ASC is proposed to detect unknown classes utilizing support vector data description (SVDD). This method is only able to detect unknown samples without being able to learn them. In [7], a real-time unsupervised model for learning environmental noise signals is developed. This model can detect unknown classes in the stream of input sound signals and learn them on the fly. However, it can only store data from one unknown class and create one class at a time.

In this work, we propose a solution to overcome the limitations of both systems. Our proposed technique can identify the unknown sound signals and classify them into multiple micro-clusters based on the similarity of their characteristics. We then prune the microclusters according to a popularity measure, such that the micro-clusters larger than a certain threshold are classified into new classes on the fly. Importantly, our proposed method has no limitation on the number of classes created on the fly.

The rest of the paper is organized as follows. A brief overview of the open-set problem is covered in Section 2. The proposed openset evolving acoustic scene classification model is then presented in Section 3. Section 4 presents the experimental results followed by the conclusion in Section 5.

\section{OPEN-SET RECOGNITION MODEL}

In this section, we first briefly state the preliminaries related to openset recognition (OSR), following which we formally define the evolving open-set problem in Section 3.

Traditional recognition/classification algorithms are closed-set problems where all training and testing data are known a priori. Closed-set classifiers have been developed that maximize the optimal posterior probability, $p\left(C_{i} \mid x ; C_{1}, C_{2}, \ldots C_{M}\right), i \in\{1,2, \ldots, M\}$, where $x$ is an input sample, $i$ is the index of the known class $C_{i}$, and $M$ in the number of the known classes. However, a practical automatic recognition/classification problem is an open-set problem, 


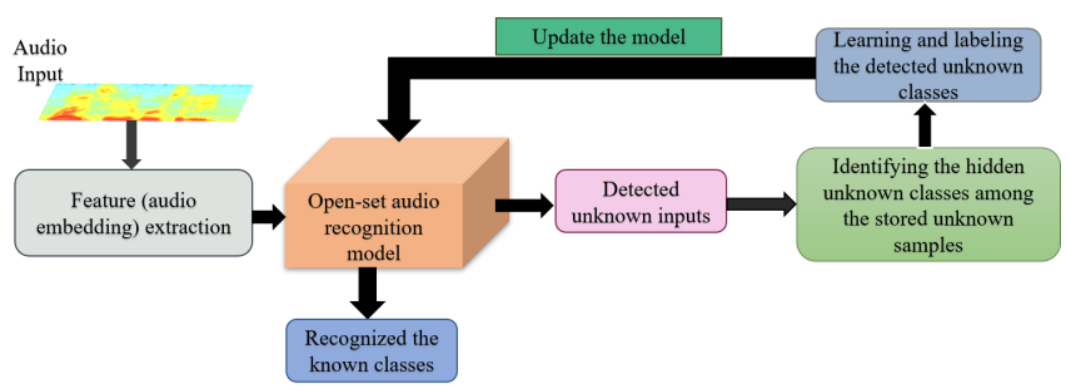

Figure 1: Block diagram of the developed open-set evolving acoustic scene classification system

where during testing, data from unknown classes can emerge at any time unexpectedly. Therefore, during the test, the optimal posterior probability becomes $p\left(C_{j} \mid x ; C_{1}, C_{2}, \ldots C_{M}, C_{M+1}, \ldots C_{M+Q}\right), j \in$ $\{1,2, \ldots, M+Q\}$, with $Q$ being the number of unknown classes [8]. This posterior probability cannot be modeled as classes $C_{M+1}$ through $C_{M+Q}$ are unknown.

In [9], the OSR problem was formalized for the first time and a preliminary solution was proposed. It incorporates an open space $O$ risk term to account for the space beyond the reasonable support of the known classes. Open space is a space that is sufficiently far from the known classes. Let $f$ be a measurable function where $f_{y}(x) \geq 0$ implies recognition of the known class $y$ and $f_{y}(x)=0$ implies, input $x$ does not belong to class $y$. The open space risk $R_{o}(f)$ for a class $y$ can be defined as follows [9]:

$$
R_{o}\left(f_{y}\right)=\frac{\int_{o} f_{y}(x) d x}{\int_{S_{o}} f_{y}(x) d x}
$$

where $S_{o}$ is the space that contains all the known training classes as well as the open space $O$. The objective OSR function must balance the open space risk against empirical error.

The OSR problem has been studied in various frameworks [1012]. In [12] the existing open-set techniques are categorized into five main categories. 1) deep neural network-based, 2) adversarial learning-based, 3) extreme value theory-based, 4) Dirichlet process-based, and 5) traditional machine learning-based models. Although much effort has been made to develop promising solutions for OSR problem, the flexibility of these methods in continuously learning new classes, at a lower computational complexity, is still a challenge. Addressing this challenge using traditional machine learning approaches is the focus of this paper.

\section{MULTI-CLASS OPEN-SET EVOLVING ACOUSTIC SCENE CLASSIFICATION MECHANISM}

A dynamically evolving classification system needs to continuously detect unknown classes and learn them at a low training cost. The key components of such a system are as follows: 1) accurately assigning the input samples from the known classes into their respective labels, 2) rejecting samples that are from unknown classes, 3) keeping track of the rejected unknown samples to identify potential new classes among all the rejected samples in 2), and 4) learning/labeling the detected new classes and expanding the existing model at a low training cost.

In this work, similar to [7], known classes are labeled with positive integers and unknown samples are temporarily labeled as 0 . Assuming $x \in \mathbb{R}^{d}$ to be an input sample in the feature domain, the proposed open-set evolving model is defined as follows.
Definition (Multi-class Open-set Evolving Recognition (MCOSR) Model): A solution to a multi-class open-set evolving recognition:

1. A multi-class open-set model $F(x): \mathbb{R}^{d} \mapsto \mathbb{Z}^{\geq 0}$ is an ensemble of multiple OSR functions, $f_{C_{i}}(x): \mathbb{R}^{d} \mapsto \mathbb{R}, \quad i=1, \ldots, M$, where $M$ is the number of known classes.

2. Let $\mathcal{X}_{\text {Nov }}:=\left\{x_{k} \mid x_{k} \in \mathbb{R}^{d}, k=1, \ldots, \mathcal{K}\right\}$ be a dataset of $\mathcal{K}$ samples, that are detected as unknown. $L\left(\chi_{\text {Nov }}\right): \mathbb{R}^{d} \mapsto \mathbb{N}$ is a novelty detection process that is applied to $\mathcal{X}_{\text {Nov }}$ to determine the existence of new classes in $X_{\text {Nov }}$.

3. If $L\left(X_{N o v}\right)$ discovers $Q$ potential new classes, the existing MCOSR model is expanded by adding the discovered classes to the previously learned class set. Thus, the set of known classes becomes $\left\{C_{1}, C_{2}, \ldots, C_{M}\right\} \cup\left\{C_{M+1}, C_{M+2}, \ldots, C_{M+Q}\right\}$. It is worth noting that $M$ varies in time as a result of the evolution process. The diagram of the proposed MCOSR is shown in Fig. 1. Details of each step are introduced in the following subsections.

\subsection{Multi-class open-set evolving recognition function}

This section states the details of the proposed open-set acoustic scene classification. The algorithm consists of four main steps, feature extraction, classification/rejection, new class detection, and model evolution as follows.

\subsubsection{Feature extraction:}

The spectrogram of the input audio signal is first passed through a pre-trained neural network (details discussed in section 4) to extract the embedding representation. In this work, an L3-Net-based [13] audio embedding network is used as the feature extractor. The extracted embeddings are used as the input feature vectors to the MCOSR model.

\subsubsection{Classification/rejection}

The extracted embeddings from an input sound file are passed into the multi-class open-set recognition model $F(x): \mathbb{R}^{d} \mapsto \mathbb{Z}^{\geq 0}$ to determine if the input sound signal belongs to any of the known classes, $C_{i}$, or it is an unknown sample (0). $F(x)$ is an ensemble of multiple OSR functions, $f_{C_{i}}(x): \mathbb{R}^{d} \mapsto \mathbb{R}, \quad i=1, \ldots, M$, where $M$ is the number of known classes. Each of the OSR functions characterizes one of the known classes utilizing a support vector data description (SVDD) model [14].

SVDD is a kernel-based sphere-shaped data description method that provides an effective description of the data boundary in the feature space. SVDD has been investigated in the context of various open-set problems $[15,16]$. The objective of SVDD is to find the 
smallest hypersphere that encloses most of the data in feature space $\mathcal{X}$. Let $\mathcal{X}:=\left\{x_{j} \mid x_{j} \in \mathbb{R}^{d}, j=1, \ldots, \mathcal{J}\right\}$ be a dataset of $\mathcal{J}$ points. Using a nonlinear transformation $\varphi$ from $\mathcal{X}$ to a high-dimensional kernel feature space, the smallest enclosing hypersphere of radius $R$ and center $\alpha$ can be stated as:

$$
\begin{aligned}
& \min _{R, \alpha, \xi} R^{2}+\frac{1}{\gamma} \sum_{j} \xi_{j} \\
& \text { s.t. }\left\|\varphi\left(x_{j}\right)-\alpha\right\|^{2} \leq R^{2}+\xi_{j}, \quad \xi_{j} \geq 0, \quad \forall j .
\end{aligned}
$$

The slack variables $\xi_{j} \geq 0$, associated with each training sample $x_{j}$, allow a soft boundary and hyperparameter $\gamma \in(0,1]$ establishes a trade-off between the sphere volume and the accuracy of data description. To optimize $\alpha, R$ and $\xi_{j}$ [14] a Lagrangian procedure is used. The local maximum of the Lagrange function can be written as:

$$
\begin{aligned}
& \mathcal{L}=\sum_{j=1}^{\mathcal{J}} \beta_{j} \varphi\left(x_{j}\right) \cdot \varphi\left(x_{j}\right)-\sum_{j, k=1}^{\mathcal{J}} \beta_{j} \beta_{k} \varphi\left(x_{j}\right) \cdot \varphi\left(x_{k}\right) \\
& \text { s.t. } \sum_{j} \beta_{j}=1, \alpha=\sum_{j} \beta_{j} \varphi\left(x_{j}\right), 0 \leq \beta_{j} \leq \gamma
\end{aligned}
$$

where $\beta_{j} \geq 0$ represent Lagrange multipliers. Samples with $\beta_{j}=0$ lie inside the sphere surface, while those with $\beta_{j}=\gamma$ fall outside. Samples with $0<\beta_{j}<\gamma$ are on the boundary of the corresponding hypersphere. It can be seen from (4), the center of the sphere $(\alpha)$ is a linear combination of the data samples. To describe the hypersphere, only samples with $\beta_{j}>0$ are needed, hence they are called support vectors. $R^{2}$ is the distance from the center of the sphere $(\alpha)$ to (any of the support vectors on) the boundary, excluding the ones outside the sphere.

Therefore, given a set of $\mathcal{J}$ data samples, the open-set recognition function for representing it as a class/hypersphere $C_{i}$ is defined as:

$$
f_{C_{i}}(x)=\left\|\varphi(x)-\alpha_{i}\right\|^{2}-R_{i}^{2}
$$

Input $x$ is associated with class $C_{i}$, if its distance to the center of the sphere $C_{i}$, i.e. $\alpha_{i}$, is equal or smaller than the radius $R_{i}^{2}$, i.e. $f_{C_{i}}(x) \leq 0$. Therefore, the decision mechanism of identifying the label of the input sample $x$ in MCOSR is as follows:

$$
C^{*}=\left\{\begin{array}{cc}
\arg \min _{i} f_{C_{i}}(x) & f_{C_{i}}(x) \leq 0, \forall i=1, \ldots, M \\
0 & \text { otherwise }
\end{array}\right.
$$

The $C^{*}$ is the output label, where 0 stands for unknown samples.

To minimize misclassifications, majority voting of 3 decisions is considered. This way if inputs at time $t$ and $t+2, x_{t}$ and $x_{t+2}$ are both from class $\ell$, then $x_{t+1}$ is expected to be from the same class, noting that signal data occur in a streaming manner and the interest is primarily on the sustained type of sound.

\subsubsection{Model evolution with detected new classes}

Samples that are detected as unknown are stored in a buffer. Length of this buffer should be larger than a minimum number of samples, $\mathcal{D}$, required for establishing a new class. Let $\mathcal{X}_{\text {Nov }}:=\left\{x_{k} \mid x_{k} \in\right.$ $\left.\mathbb{R}^{d}, k=1, \ldots, \mathcal{K}\right\}$ be the set of $\mathcal{K}$ samples in the buffer. Among these stored unknown samples, we need to determine if there is any consolidated ensemble that should be declared as a new separate class. A similarity measure is used to assess such consolidation. Let $L():. \mathbb{R}^{d} \mapsto \mathbb{N}$ to be the process of detecting the existence of a new class using that similarity measure. We use cosine similarity measure and denote a pair of data points as similar if their cosine similarity is greater than a predefined threshold value $\lambda$. In this work, this value is set empirically to $\lambda=0.85$. An ensemble of similar samples is called micro-cluster. A sample is assigned to a microcluster if its similarity not only with the center of the micro-cluster, but also with each sample from a randomly selected set, is greater than $\lambda$. If not assigned to any of the existing micro-clusters, the sample will form a sporadic micro-cluster. Micro-clusters with size less than two are considered sporadic. Once the size of a micro-cluster exceeds $\mathcal{D}$, an open-set recognition function, e.g. SVDD, is used to model it as a new class. To set $\mathcal{D}$, one needs to specify how long an occurring sound to be sustained in order to establish a new class. In other words, how long sound data from a scene class is needed to establish a new class. Finally, when a new class is created, the pretrained model gets updated accordingly. The newly created class is labeled as the number of existing classes plus one.

\section{EXPERIMENTAL RESULTS}

In this section, we evaluate the performance of MCOSR algorithm in terms of OSR accuracy, and qualitatively analyze its evolving aspects. We then perform a comparative study of the OSR accuracy of the proposed method against the existing state-of-the-art OSR algorithms such as EVM [8], Weibull-calibrated SVM (W-SVM) [17], PI-SVM and PI-OSVM (one-class SVM) [18]. EVM has a well-grounded interpretation derived from the statistical extreme value theory (EVT) and is the first classifier capable of performing nonlinear kernel-free learning. In W-SVM, the decision scores are used to fit the data into a single Weibull distribution, and a specific threshold is set to reject the unknown classes [8].

Data from the DCASE 2018 task5, a subset of SINS [19], TUT Acoustic Scenes 2017 [20], and Audioset [21] are used to evaluate the proposed algorithm. The dataset comprises 12 acoustic scenes, which are "absence", "cooking", "dishwashing", "eating", "social activity", "watching TV", “car", "music", "restaurant”, "transport”, "vacuum cleaner", and "working".

OpenL3 [22] is used for extracting the audio embeddings, considering the default settings and a 512-embedding dimension. The input to the L3-Net is a 10-second audio signal and the extracted embedding feature is a matrix of size $96 \times 512$. The averages of the extracted embeddings from the 10 -second sound files are used as inputs to the MCOSR.

We begin by pre-training a model using data from the following five acoustic scenes "cooking", "dishwashing", "eating", "social activity", and "watching TV". This leads to five pre-trained classes, which we will refer to as $\mathrm{C} 1, \mathrm{C} 2, \ldots, \mathrm{C} 5$, respectively. For pre-training, we used 30 audio embedding samples, i.e. $\mathcal{D}$, extracted from five minutes of sound signals per acoustic scene. The remaining duration of data associated with the five pre-training scenes, mentioned above, along with the full duration of the data associated with the other seven acoustic scenes, i.e. "absence", "car", "music", "restaurant", "transport", "vacuum cleaner", and "working", are used for testing.

\subsection{Multi-class open-set recognition}

We evaluate the OSR aspect of the MCOSR in two steps. In the first step, the proposed system identifies if an input sample is associated with the pre-trained classes and if so, it labels them as known samples. In the second step, the system classifies the known samples into their respective classes, which is known as closed-set recognition. Denote by $T P, F P, T N$, and $F N$ the true-positive, false-positive, true-negative and false-negative, respectively. We measure the 
Table 1. Accuracy of MCOSR, EVM, W-SVM(Linear), PIOSVM, and PI-SVM in detecting the known classes and rejecting the unknown classes (\%), in terms of TPR (higher, better) and 1-FPR (higher, better)

\begin{tabular}{ccc}
\hline Methods & $\begin{array}{c}\text { Detecting known: } \\
\text { TPR }\end{array}$ & $\begin{array}{c}\text { Rejecting unknown: } \\
\text { 1-FPR }\end{array}$ \\
\hline \hline MCOSR & $\mathbf{8 8 . 2 2}$ & $\mathbf{9 3 . 0 3}$ \\
\hline W-SVM (Linear) & $\mathbf{5 9 . 5 8}$ & $\mathbf{9 6 . 6 1}$ \\
\hline EVM & $\mathbf{9 7 . 0 8}$ & $\mathbf{9 1 . 9}$ \\
\hline PI-OSVM & 21.7 & 15.68 \\
\hline PI-SVM & 60.8 & 51.41 \\
\hline
\end{tabular}

accuracy of the first step in the form of true-positive rate, given by $T P R=T P /(T P+F N)$, and specificity, that is $1-F P R=T N /$ $(T N+F P)$. The results are reported in Table 1, where each entry is an average of a 10 -fold cross-validation process.

Aside from MCOSR results, Table 1 also reports the results for comparable algorithms in the literature, including EVM, W-SVM, PI-SVM, and PI-OSVM. The rejection threshold, i.e. $\delta$, for EVM is set to 0.05 , which is deduced empirically. At first glance, it may appear that W-SVM outperforms other algorithms since its specificity is slightly higher than that of MCOSR and EVM. However, it drastically underperforms, in terms of $T P R$, compared to MCOSR and EVM. It is worth mentioning that, EVM has the highest TPR while its specificity is close to the MCOSR. Importantly, as reported in Table 2, EVM suffers from large confusion error in close-set recognition. Our analyses suggest that PI-OSVM and PI-SVM provide the lowest performance.

\subsection{The performance of the open-set technique as a function of class-set evolution}

In this section, we study the performance of our proposed algorithm while changing the order in which the data are fed to the system. Our contribution is two-fold. First, we investigate the effect of choosing the initial sound samples on the number of created classes during the test and their accuracy. Next, we measure the overall post-evolution accuracy of the system, in terms of a confusion matrix.

To evaluate the effect of the initial sound samples on the performance of the proposed MCOSR technique, we used a pre-trained model comprising $\mathrm{C} 1, \mathrm{C} 2, \mathrm{C} 3, \mathrm{C} 4$, and $\mathrm{C} 5$. During the test, the inputs to the pre-trained model are the samples from known and unknown classes. The experiment is repeated 10 times while randomly shuffling the sound files associated with each class. Samples that are detected as unknown are stored in a buffer. The $L($.$) process is$ continuously assessing the existence of a new class among the stored unknown samples. Once it detects a new class, MCOSR to be updated with this new class and the set of known classes is expanded. It is expected the forthcoming samples from the newly added class to be assigned to it and not identified as unknown samples anymore. However, in some of the experiments, MCOSR creates multiple classes for "music" class. Because the music data is not coherent enough, the initially created music class by MCOSR does not provide enough representative information of the music class. Therefore, other incoming music samples are identified as unknown samples and later will be learned as a new class. Data from the "working" sound scene is also a challenging one. In some experiments, no new class is created for this scene, as data from this scene is always misclassified by one of the known classes, mostly the "absence" class. Also, the samples from this scene that are identified as unknown are not similar enough to create meaningful micro-clusters to be declared as a new class.

As mentioned earlier, we next measured the post-evolution accuracy of the system. The results are reported in Table 3 in terms of a confusion matrix. In this table "absence", "car", "music", "restaurant", "transport", "vacuum cleaner", and "working" scenes are referred as $\mathrm{C} 6, \mathrm{C} 7, \ldots, \mathrm{C} 12$, respectively. It was found as the number of classes increases in the model; the confusion rate increases. Because SVDD ignores the discriminative information between the known classes, which leads to poor classification performance. This issue needs to be addressed to achieve a higher classification accuracy when dealing with more complex acoustic scenes.

\section{CONCLUSION}

This paper provides an open-set evolving audio scene classification technique, MCOSR, which can effectively recognize and learn unknown acoustic scenes in an unsupervised manner. The developed model is evaluated utilizing the DCASE challenge dataset, TUT Acoustic Scenes 2017, and music files from Audioset. Experimental results demonstrate the effectiveness of the developed approach in identifying unknown samples compared to EVM, W-SVM, PIOSVM, and PI-SVM. This paper exemplifies how the proposed MCOSR method can be used as a proper evolving open-set system for sound classification applications. Future research will focus on addressing practical issues during run time.

Table 2. Confusion matrices of the proposed MCOSR and EVM for Table 1.

\begin{tabular}{|c|c|c|c|c|c|c|c|c|c|c|c|c|}
\hline & \multicolumn{2}{|c|}{ cooking } & \multicolumn{2}{|c|}{ dishwashing } & \multicolumn{2}{|c|}{ eating } & \multicolumn{2}{|c|}{ social activity } & \multicolumn{2}{|c|}{ watching TV } & \multicolumn{2}{|c|}{ unknown } \\
\hline & MCOSR & EVM & MCOSR & EVM & MCOSR & EVM & MCOSR & EVM & MCOSR & EVM & MCOSR & EVM \\
\hline cooking & 81.54 & $\mathbf{5 8 . 3 3}$ & 3.97 & 27.08 & 0 & 4.17 & 0 & 0 & 0 & 0 & 14.49 & 10.42 \\
\hline dishwashing & 3.08 & 16.67 & 88.21 & 62.5 & 3.72 & 20.83 & 0 & 0 & 0 & 0 & 5 & 0 \\
\hline eating & 0 & 8.33 & 1.03 & 12.5 & 96.41 & 79.17 & 0 & 0 & 0 & 0 & 2.56 & 0 \\
\hline social activity & 0 & 6.25 & 0 & 0 & 1.28 & 4.17 & 86.28 & 87.5 & 0 & 0 & 12.44 & 2.08 \\
\hline watching TV & 0.13 & 2.08 & 0 & 0 & 0 & 2.08 & $\begin{array}{l}0.9 \\
\end{array}$ & 6.25 & 89.1 & 87.5 & 9.87 & 2.08 \\
\hline unknown & 0.04 & 7.79 & 0.04 & 0.31 & 5.48 & 0 & 0 & 0 & 0.15 & 0 & 94.29 & 91.9 \\
\hline
\end{tabular}

Table 3. Accuracy of the developed open-set evolving acoustic scene recognition model (\%), after all classes have been learnt on the fly

\begin{tabular}{|c|c|c|c|c|c|c|c|c|c|c|c|c|c|}
\hline & $\mathrm{C} 1$ & $\mathrm{C} 2$ & $\mathrm{C} 3$ & $\mathrm{C} 4$ & $\mathrm{C} 5$ & C6 & $\mathrm{C} 7$ & C8 & C9 & $\mathrm{C} 10$ & $\mathrm{C} 11$ & $\mathrm{C} 12$ & unknown \\
\hline $\mathrm{C} 1$ & $\begin{array}{l}73.21 \\
\end{array}$ & " & 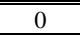 & 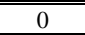 & 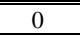 & 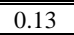 & $\begin{array}{c}1.03 \\
\end{array}$ & 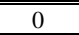 & 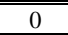 & $\begin{array}{c}0 \\
\end{array}$ & $\begin{array}{c}0 \\
\end{array}$ & 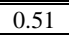 & 20.77 \\
\hline $\mathrm{C} 2$ & 2.05 & 88.46 & 3.08 & 0 & 0 & 0 & 0 & 0 & 0 & 0 & 0 & 0 & 6.41 \\
\hline $\mathrm{C} 3$ & 0 & 1.67 & 81.67 & 0 & 0 & 4.36 & 0 & 0 & 0 & 0 & 0 & 3.08 & 9.23 \\
\hline $\mathrm{C} 4$ & 0.13 & 0.38 & 0.51 & 84.62 & 0 & 0 & 0 & 0 & 1.15 & 0.38 & 0 & 0 & 12.82 \\
\hline $\mathrm{C} 5$ & 0 & 0 & 0 & 0.13 & 89.74 & 0 & 0 & 0 & 0 & 0 & 0.51 & 0.51 & 9.1 \\
\hline C6 & 0.13 & 0 & 0.26 & 0 & 0 & 94.49 & 0 & 0 & 0 & 0 & 0 & 0.13 & 5 \\
\hline $\mathrm{C} 7$ & 0 & 0 & 0 & 0 & 0 & 1.28 & 92.95 & 0 & 0 & 0.26 & 0 & 0 & 5.51 \\
\hline $\mathrm{C} 8$ & 0 & 0 & 0.9 & 0 & 0 & 0 & 0 & 94.62 & 0.64 & 0 & 0 & 0 & 3.85 \\
\hline C9 & 0 & 0 & 0 & 0 & 0 & 0 & 0 & $\begin{array}{l}0.9 \\
\end{array}$ & 97.56 & 0 & 0 & 0 & 1.54 \\
\hline $\mathrm{C} 10$ & 0 & 0 & 0 & 0.77 & 0 & 0 & 0.26 & 0 & 0 & 92.56 & 0 & 0 & 6.41 \\
\hline C11 & 0 & 0 & 0 & 0 & 0 & 0 & 0 & 0 & 0 & 0.77 & 91.15 & 0 & 8.08 \\
\hline $\mathrm{C} 12$ & 0.63 & 2.7 & 13.81 & 0 & 0.63 & 29.05 & 0 & 0 & 0 & 0 & 0 & 19.68 & 33.49 \\
\hline
\end{tabular}




\section{REFERENCES}

[1] S. McAdams, "Recognition of sound sources and events," in Thinking in Sound,: the Cognitive Psychology of Human Audition. London: Oxford Univ. Press, 1993.

[2] A. S. Bregman. Auditory Scene Analysis: The Perceptual Organization of Sound. Cambridge, MA, USA: MIT Press, 1990.

[3] I. Panahi, N. Kehtarnavaz, and L. Thibodeau, "Smartphonebased noise adaptive speech enhancement for hearing aid applications," Proceedings of the $38^{\text {th }}$ Annual International Conference of the IEEE Engineering in Medicine and Biology Society $(E M B C)$, pp. 85-88, Orlando, August 2016.

[4] S. Aziz, M. Awais, T. Akram, U. Khan, M. Alhussein, and K. Aurangzeb, "Automatic scene recognition through acoustic classification for behavioral robotics," Electronics 2019, vol. 8, no.5, 483 .

[5] T .Virtanen, M. D. Plumbley, and D. Ellis. "Introduction to sound scene and event analysis," Computational Analysis of Sound Scenes and Events, pp. 3-12, Springer, Cham, 2018.

[6] D. Battaglino, L. Lepauloux, and N. Evans, "The open-set problem in acoustic scene classification," International Workshop on Acoustic Signal Enhancement (IWAENC), pp. 1-5, 2016.

[7] F. Saki, N. Kehtarnavaz "Real-time unsupervised classification of environmental noise signals," IEEE/ACM Transactions on Audio, Speech, and Language Processing, pp. 1657-1567, 2017.

[8] E. M. Rudd, L. P. Jain, W. J. Scheirer, and T. E. Boult, "The extreme value machine," IEEE Transactions on Pattern Analysis and Machine Intelligence, vol. 40, no. 3, pp. 762-768, Mar. 2018.

[9] W. J. Scheirer, A. de Rezende Rocha, A. Sapkota, and T. E. Boult, "Toward open set recognition," IEEE Transactions on Pattern Analysis and Machine Intelligence, vol. 35, no. 7, pp. 1757-1772, Jul. 2013.

[10] P. Phillips, P. Grother, and R. Micheals, "Evaluation methods on face recognition," Handbook of Face Recognition, A. Jain and S. Li, eds., pp. 329-348, Springer, 2005.

[11] L. Fayin and H. Wechsler, "Open set face recognition using transduction," IEEE Transactions on Pattern Analysis and Machine Intelligence, vol. 27, no. 11, pp. 1686-1697, Nov. 2005.

[12] Ch. Geng, Sh. Huang, and S. Chen, "Recent advances in open set recognition: a survey," http://arxiv.org/abs/1811.08581.

[13] R. Arandjelovi'c and A. Zisserman, "Look, listen and learn," Proceedings of the IEEE International Conference on Computer Vision (ICCV), pp. 609-617, 2017.

[14] D. M. Tax and R. P. Duin, "Support vector data description," Machine learning, vol. 54, no. 1, pp. 45-66, 2004.

[15] A. Banerjee, P. Burlina, and C. Diehl. "A support vector method for anomaly detection in hyperspectral imagery," IEEE Transactions on Geoscience and Remote Sensing, vol. 44, no. 8, pp. 2282-2291, 2006.

[16] F. Alegre, A. Amehraye, and N. Evans. "A one-class classification approach to generalised speaker verification spoofing countermeasures using local binary patterns," In Proceedings of the IEEE Sixth International Conference on Biometrics: Theory, Applications and Systems (BTAS), pp. 1-8, 2013.

[17] W. J. Scheirer, L. P. Jain, and T. E. Boult, "Probability models for open set recognition," IEEE Transactions on Pattern Analysis and Machine Intelligence, vol. 36, no. 11, pp. 2317-2324, 2014.
[18] L. P. Jain, W. J. Scheirer, and T. E. Boult, "Multi-class open set recognition using probability of inclusion," European Conference on Computer Vision, pp. 393-409, Springer, Cham, 2014.

[19] G. Dekkers, S. Lauwereins, B. Thoen, M. W. Adhana, H Brouckxon, T. van Waterschoot, B. Vanrumste, M. Verhelst, and P. a. Karsmakers, "The SINS database for detection of daily activities in a home environment using an acoustic sensor network," Proceedings of the Detection and Classification of Acoustic Scenes and Events 2017 Workshop (DCASE2017), November 2017.

[20] A. Mesaros, T. Heittola, and T. Virtanen. TUT database for acoustic scene classification and sound event detection. In $24^{\text {th }}$ European Signal Processing Conference, pp.1128-1132, Budapest, Hungary, 2016.

[21] J. F. Gemmeke, D. P. W. Ellis, D. Freedman, A. Jansen, W. Lawrence, R. C. Moore, M. Plakal, and M. Ritter, "Audio Set: An ontology and human-labeled dataset for audio events," Proceedings of the EEE International Conference on Acoustics, Speech and Signal Processing (ICASSP), pp.776-780, 2017.

[22] J. Cramer, H. Wu, J. Salamon, and J. Pablo Bello "Look, listen and learn more: design choices for deep audio embeddings," Proceedings of the IEEE International Conference on Acoustics, Speech and Signal Processing (ICASSP), pp. 3852-3856, Brighton, UK, May 2019. 